Mathematical Modelling and Analysis

Volume 19 Number 3, June 2014, 347-358

http://dx.doi.org/10.3846/13926292.2014.924444

(c) Vilnius Gediminas Technical University, 2014
Publisher: Taylor\&Francis and VGTU

http://www.tandfonline.com/TMMA

Print ISSN: 1392-6292

Online ISSN: 1648-3510

\title{
Extraction of Molasses from Sugar Crystals in a Centrifuge
}

Colin P. Please ${ }^{a}$, Neville D. Fowkes ${ }^{b}$, David P. Mason ${ }^{c}$, Masood M. Khalique ${ }^{d}$, Ashleigh Jane Hutchinson ${ }^{c}$, Maryke Rademeyer ${ }^{c}$, Richard Loubser $^{e}$ and Steve Davis ${ }^{e}$

${ }^{a}$ University of Oxford

Oxford, United Kingdom

${ }^{b}$ University of Western Australia

Crawley, WA 6009 Perth, Australia

${ }^{c}$ University of the Witwatersrand

Private Bag 3, Wits 2050 Johannesburg, South Africa

${ }^{d}$ North West University

Private Bag X2046, 2735 Mmabatho, South Africa

e Sugar Milling Research Institute

Durban, South Africa

E-mail: please@maths.ox.ac.uk

E-mail: neville.fowkes@uwa.edu.au

E-mail: David.Mason@wits.ac.za

E-mail: Masood.Khalique@nwu.ac.za

E-mail(corresp.): hutchinson. ash@gmail.com

E-mail: maryke.rademeyer@students.wits.ac.za

E-mail: rloubser@smri.org

E-mail: sdavis@smri.org

Received April 9, 2013; revised April 21, 2014; published online June 1, 2014

\begin{abstract}
Massecuite is a mixture of sugar crystals and molasses produced during the manufacture of sugar. A centrifuge, which is a rotating cylindrical basket, is used to separate the sugar crystals from the molasses. Water and steam are introduced into the centrifuge during the latter part of the process to further facilitate drainage. Models developed indicate that a fifty percent increase in molasses drainage can result from the addition of steam, whereas water does not significantly affect drainage.
\end{abstract}

Keywords: mathematical model, porous media, differential equation.

AMS Subject Classification: $76 \mathrm{~S} 05$. 


\section{Introduction}

The process of extracting the liquid component of the massecuite, called molasses, from sugar crystals is not optimal. In the early stages of the extraction process, a significant percentage of molasses is not drained from the centrifuge and more time is required to complete the procedure. Currently, the addition of water or steam is used to aid in the efficiency of this process, which was investigated by Jullienne [9]. Hydrogen peroxide may also be added to act as a bleach to reduce the colour of the sugar. Results for this method are presented by Saska [11]. In this paper, we use a simple mathematical model in order to investigate the mechanism of this extraction process. We show that the process can be modified to improve the drainage of molasses from the sugar crystals and thus decrease the time required for its completion. According to the model, the addition of steam leads to improving the drainage of molasses by about fifty percent.

During the first stage of the sugar manufacturing process juice is extracted from shredded sugar cane in a diffuser. The concentration of sugar is then increased to the point of supersaturation by evaporation. The resulting substance termed massecuite consists of roughly $50 \%$ sugar crystals suspended in the molasses which is a non-Newtonian fluid. The combination has an average viscosity $0.8 \mathrm{~Pa}$.sec. The massecuite is deposited into a batch centrifuge consisting of a cylindrical basket which is spun at high speed so that the liquid surrounding the crystals, now referred to as molasses, is extracted through the walls of the basket as shown in Figure 1. A detailed description of the development of the centrifuge in the sugar manufacture process has been given by Davidenko et al. [4]. The basket has a diameter of $1.2 \mathrm{~m}$ and is lined by a fine mesh which allows for the passage of liquid but ensures that the crystals remain trapped inside.

While the massecuite is being deposited into the basket, the rotational velocity of the centrifuge is about $200 \mathrm{rev} / \mathrm{min}$. The massecuite that is fed into the bottom of the centrifuge first spreads out in the radial direction and then creeps up the insides of the basket. Alternatively the massecuite may be sprayed directly onto the basket. During this stage of the process the mixture behaves like a homogeneous viscous liquid [6]. After some time the rotational velocity of the centrifuge is increased to $960 \mathrm{rev} / \mathrm{min}$ which it then maintains. By this stage a layer of massecuite of about $100 \mathrm{~mm}$ has built up on the interior vertical sides of the basket. This is known as the spin up stage of the extraction process. The effects of speed on the quality of the sugar produced is considered by Bullen and de Bruijn [3]. Once the centrifuge reaches a constant angular velocity, the molasses filters through the crystal matrix formed against the walls of the basket. For sufficiently low liquid concentration levels, the flow of molasses through the wall of the centrifuge essentially ceases and the remaining liquid is flushed out from the crystals via the addition of water or steam. After molasses 'removal' the centrifuge is slowed down and the sugar crystals are removed by a plough-like mechanism. The addition of water and the washing off of the molasses regulates the colour of the crystals. Further bleaching may be achieved by means of an oxidising agent such as hydrogen peroxide. 
This investigation was prompted by difficulties experienced in the sugar industry during this process. Under sub-optimal loading conditions for either the composition of the massecuite, its feeding rate, or the spin up process, the mixture will not uniformly coat the basket and this can lead to centrifuge instabilities either immediately or later during the spin cycle when spin speeds are large. These instabilities were studied, for example, by Joseph et al. [8]. Nonuniform drainage can also result and blockages may occur. During the molasses stripping process, additional difficulties can also arise during the water and steam addition stage of the process in that nonuniform washing can result in quality degradation and the dissolution of crystals. Based on experience and simple models, practical measures have been adopted to deal with these difficulties. However it is hoped that a better understanding of the processes might lead to better operational procedures. A useful reference to similar problems (but in a geophysical context) is Greenspan [6]. The parameter range is somewhat different in the present context and there are other two phase/lubrication issues that play a role. Our main concern in this paper is the extraction of molasses from the sugar crystals once the flow of molasses has basically stopped and the centrifuge has maintained a constant angular velocity for a sufficient length of time. Water or steam is required to facilitate further molasses removal in this stripping stage of the extraction process. In Sections 2 to 5 a simple model for the molasses stripping process is examined. Conclusions and suggestions are presented in Section 6.
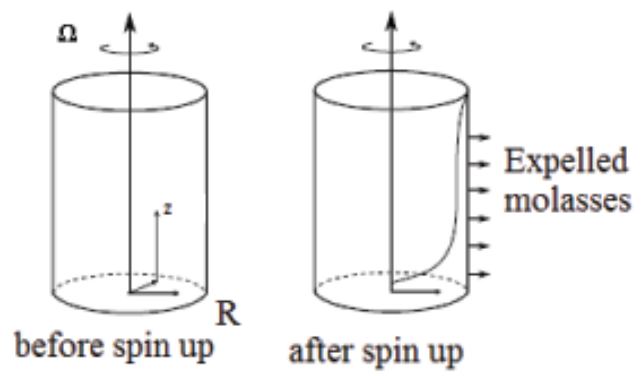

Figure 1. Cylindrical polar coordinate system $(r, \theta, z)$ for a thin film of molasses draining from sugar crystals. The coordinate system moves with the centrifuge rotating with angular velocity $\Omega$.

\section{Model Description}

When considering the problem of removing molasses from the crystals, the theory of thin fluid films applies. The model for the drainage of molasses from the sugar crystals is illustrated in Figure 2. It is assumed that the crystals form a flat wall with initially a uniform layer of molasses. It is also assumed that the molasses can flow freely through the outer wall of the centrifuge. The outer wall is made of a fine mesh which permits the passage of liquid but not the 


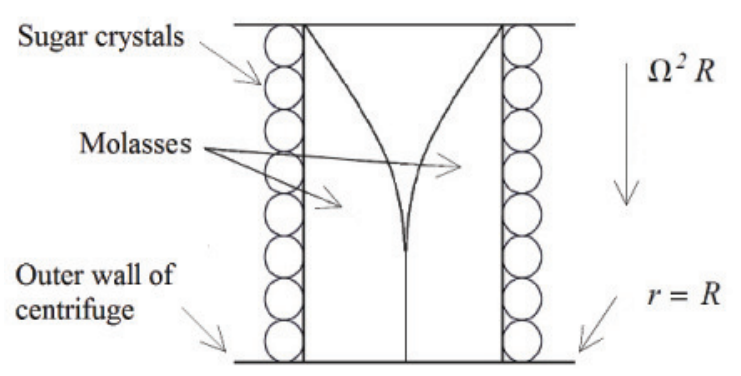

Figure 2. Drainage of molasses from crystals.

sugar crystals. The initial thickness of the molasses layer is of order $2 \times 10^{-4} \mathrm{~m}$. In Figure 2 the horizontal length scale is exaggerated for clarity. The model fixes the sugar crystals in the form of a wall of crystals of height $H=10 \mathrm{~cm}$. The molasses forms a thin fluid film on the crystal wall which drains from the surface. The flow is driven by the centrifugal force of magnitude $\Omega^{2} R$ per unit mass where $\Omega$ is the angular velocity of the centrifuge and $R$ is its radius. The drainage of molasses is a coating flow problem and thin fluid film theory may be applied. Although the molasses is a non-Newtonian fluid it will be modelled as a highly viscous Newtonian fluid.

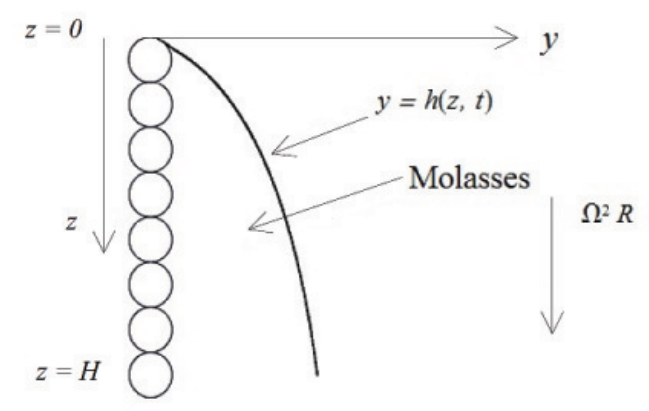

Figure 3. Coordinate system for a thin film of molasses draining from sugar crystals.

The rectangular coordinate system $(x, y, z)$ is shown in Figure 3 with $x$ measured into the page. The flow is assumed to be two-dimensional and there is therefore no dependence on $x$. The equation of the free surface of the molasses is $y=h(z, t)$. The components of the fluid velocity are

$$
v_{x}=0, \quad v_{y}=u(y, z, t), \quad v_{z}=w(y, z, t) .
$$

For the drainage problem considered here the fluid pressure is constant. The fluid sticks to the wall and there is no shear force on the surface of the molasses, 
$y=h(z, t)$. The boundary conditions are

$$
w(0, z, t)=0, \quad \frac{\partial w}{\partial y}(h(z, t), z, t)=0
$$

In the lubrication approximation of thin fluid film theory, the inertia term is neglected. The viscous force and the centrifugal force therefore balance. The thin film equation for the drainage of molasses from the crystal wall under the centrifugal acceleration $\Omega^{2} R$ is the same as that describing the two-dimensional drainage of a thin layer of viscous fluid down a vertical wall due to gravity if $g$ is replaced by $\Omega^{2} R[1]$. Hence

$$
\mu \frac{\partial^{2} w}{\partial y^{2}}=-\rho \Omega^{2} R
$$

Integrating (2.2) twice with respect to $y$ and imposing the boundary conditions in $(2.1)$, we obtain

$$
w(y, z, t)=\frac{\rho \Omega^{2} R}{2 \mu} y(2 h-y) .
$$

Now, by considering the conservation of mass equation and the free surface boundary condition it can be shown that

$$
\frac{\partial h}{\partial t}+\frac{\partial}{\partial z}(h\langle w\rangle)=0
$$

where

$$
\langle w\rangle=\frac{1}{h} \int_{0}^{h} w(y, z, t) \mathrm{d} y .
$$

Using (2.3) it follows that $\langle w\rangle=\rho \Omega^{2} R h^{2} /(3 \mu)$ and therefore

$$
\frac{\partial h}{\partial t}+\frac{\rho \Omega^{2} R}{\mu} h^{2} \frac{\partial h}{\partial z}=0 .
$$

This equation corresponds to the first order nonlinear partial differential equation found in [1] if $\Omega^{2} R$ is replaced by $g$.

In order to non-dimensionalise (2.4) we define:

$$
h=h_{0} \bar{h}, \quad t=T \bar{t}, \quad z=H \bar{z},
$$

where $h_{0}$ is the initial width of the thin film and the characteristic time $T$ is to be determined. When (2.5) is substituted into (2.4) we obtain

$$
\frac{\partial \bar{h}}{\partial \bar{t}}+\frac{\rho \Omega^{2} R h_{0}^{2} T}{\mu H} \bar{h}^{2} \frac{\partial \bar{h}}{\partial \bar{z}}=0
$$

and the initial conditions become,

$$
\begin{aligned}
& \bar{t}=0, \quad \bar{z}=0: \quad 0 \leq \bar{h} \leq 1, \\
& \bar{t}=0, \quad 0<\bar{z} \leq 1: \quad \bar{h}=1 .
\end{aligned}
$$




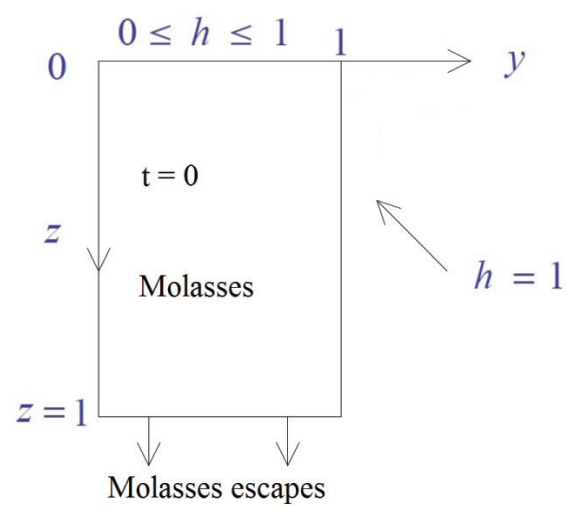

Figure 4. The initial conditions (3.2) and (3.3) for the partial differential equation (3.1).

The initial conditions (2.7), (2.8) are illustrated in Figure 4, where the overhead bars have been suppressed. These conditions assume that there is initially a uniform layer of molasses on the sugar crystals. The molasses escapes from the centrifuge for $z>1$. The initial profile is not differentiable at the point $z=0, h=1$. This point propagates forward in time and persists until time $\bar{t}=1$ as shown in Figure 5 .

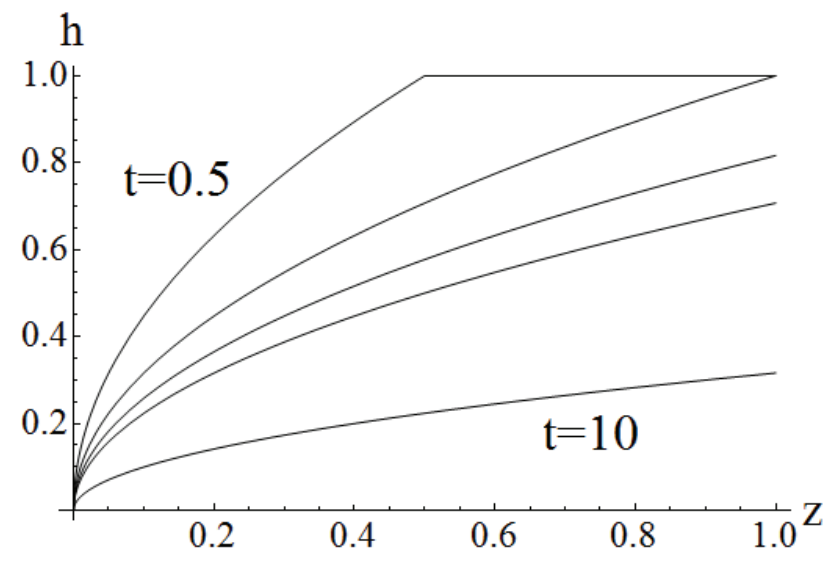

Figure 5. Graph of $h(z, t)$ against $z$ for $t=0.5,1,1.5,2$ and 10 .

The characteristic time, $T$, which is an estimate of the time taken for the molasses to drain from the sugar crystals, is therefore

$$
T=\frac{\mu H}{\rho \Omega^{2} R h_{0}^{2}} .
$$

Using (2.9), (2.6) becomes

$$
\frac{\partial \bar{h}}{\partial \bar{t}}+\bar{h}^{2} \frac{\partial \bar{h}}{\partial \bar{z}}=0
$$


We will suppress the overhead bars in the subsequent analysis it being understood that dimensionless variables are being used.

Consider now an estimate of the characteristic time $T$. For the centrifuge an estimate of the parameters in $(2.9)$ at $60{ }^{\circ} \mathrm{C}$ is:

$$
\begin{aligned}
& h_{0}=2 \times 10^{-4} \mathrm{~m}, \quad \rho=2 \times 10^{3} \mathrm{~kg} / \mathrm{m}^{3}, \quad H=0.1 \mathrm{~m}, \\
& R=0.5 \mathrm{~m}, \quad \mu=100 \text { Pas }, \quad \Omega=\frac{100 \pi}{3} \text { radians } / \mathrm{sec} .
\end{aligned}
$$

With these values of the parameters, $T=25 \mathrm{sec}$. This estimate for $T$ is within the expected range of values for the characteristic time for filtration.

\section{Mathematical Solution}

Consider the model of drainage of molasses from sugar crystals as shown in Figure 3. The thickness $h(z, t)$ of the molasses between $z=0$ and $z=1$ is described by the first order quasi-linear partial differential equation

$$
\frac{\partial h}{\partial t}+h^{2} \frac{\partial h}{\partial z}=0
$$

subject to the initial conditions

$$
\begin{aligned}
& t=0, \quad z=0: \quad 0 \leq h \leq 1 \\
& t=0, \quad 0<z \leq 1: \quad h=1
\end{aligned}
$$

We will see that we obtain a smooth solution of (3.1) which evolves from the non-differentiable initial conditions (3.2) and (3.3). The differential equations of the characteristic curves of (3.1) are

$$
\frac{d t}{1}=\frac{d z}{h^{2}}=\frac{d h}{0}
$$

and the characteristic curves are thus

$$
h=c_{1}, \quad z-c_{1}^{2} t=c_{2},
$$

where $c_{1}$ and $c_{2}$ are constants. The general solution of (3.1) is

$$
h=F\left(z-h^{2} t\right),
$$

where $F$ is an arbitrary function. Consider first $0<t \leq 1$. From the initial condition (3.3)

$$
F(z)=1, \quad 0<z \leq 1
$$

and therefore

$$
h(z, t)=1, \quad t<z \leq 1 .
$$

To determine the solution for $0 \leq t \leq z$, consider the initial condition (3.2). From (3.4), $c_{2}=0$ and since $0 \leq h \leq 1$,

$$
h(z, t)=\left[\frac{z}{t}\right]^{\frac{1}{2}}, \quad 0 \leq z \leq t .
$$


For $t>1$,

$$
h(z, t)=\left[\frac{z}{t}\right]^{\frac{1}{2}}, \quad 0 \leq z \leq 1 .
$$

From the solutions in (3.7) and (3.8) we deduce that the non-differentiable point $z=t$ evolves with the solution and the solution becomes differentiable everywhere for $t>1$. The spatial gradient is infinite at $z=0$ :

$$
\frac{\partial h}{\partial z}(z, t)=\frac{1}{2(z t)^{\frac{1}{2}}} \rightarrow \infty \quad \text { as } z \rightarrow 0, t>0 .
$$

Graphs of $h(z, t)$ plotted against $z(0 \leq z \leq 1)$ for a range of values of $t$ are presented in Figure 5. The graphs illustrate the evolution of the thickness of the layer of molasses on the crystals. For $t>1$ the thickness of the layer of the molasses on the crystals is less than unity and channels start to form in the molasses which is illustrated in Figure 5. Since this first occurs at $t=1$, it shows that the time $T$ defined by (2.9) is a suitable characteristic time for drainage of the molasses.

\section{Analysis of Results}

The objective is to drain as much molasses as possible from the sugar crystals in the least amount of time. Let $V(t)$ be the volume of the molasses remaining on the crystal wall per unit width after draining for time $t$. The volume $V(t)$ is a useful measure of the efficiency of the draining process. The cases $0<t \leq 1$ and $t>1$ need to be treated separately. For $0<t \leq 1$,

$$
V(t)=\int_{0}^{t} h(z, t) d z+\int_{t}^{1} h(z, t) d z,
$$

and using (3.6) and (3.7) we obtain

$$
V(t)=\int_{0}^{t} \frac{\sqrt{z}}{\sqrt{t}} d z+\int_{t}^{1} 1 d z
$$

and thus $V(t)=1-\frac{1}{3} t$. Also,

$$
\frac{d V}{d t}=-\frac{1}{3}
$$

For $t \geq 1$ and using (3.8),

$$
V(t)=\int_{0}^{1} \frac{\sqrt{z}}{\sqrt{t}} d z
$$

which simplifies to $V=\frac{2}{3 \sqrt{t}}$ and we have

$$
\frac{d V}{d t}=-\frac{1}{3 t^{\frac{3}{2}}}
$$




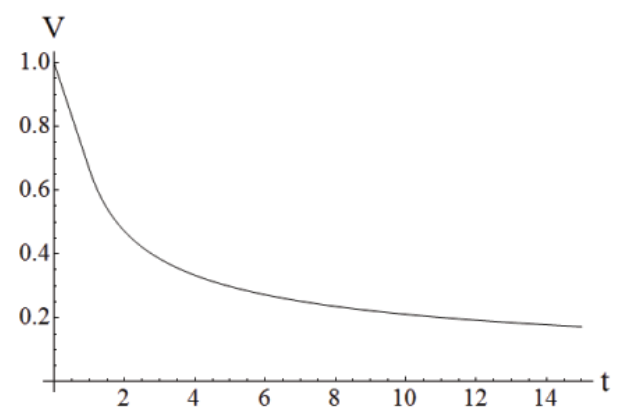

Figure 6. Graph of the volume remaining, $V(t)$, plotted against drainage time $t$.

From (4.1) and (4.2) we see that the molasses drains rapidly and at a constant rate for $0<t \leq 1$ but for $t>1$ the rate of drainage decreases. A graph of the volume remaining, $V$, plotted against $t$ is shown in Figure 6. After draining for time $t=15$, approximately $20 \%$ of molasses is not drained and therefore this percentage of molasses is not extracted.

Consider now the thickness of the molasses layer on the crystals. At $z=1$, $h(1, t)$ provides an indication of the spacing between adjacent fluid segments at the wall of the centrifuge as shown in Figure 1. It gives a measure of the growth of the channelling in the molasses. From (3.6) and (3.8)

$$
0<t \leq 1: \quad h(1, t)=1, \quad t \geq 1: \quad h(1, t)=\frac{1}{\sqrt{t}} .
$$

The graph of $h(1, t)$ plotted against time $t$ is shown in Figure 7 .

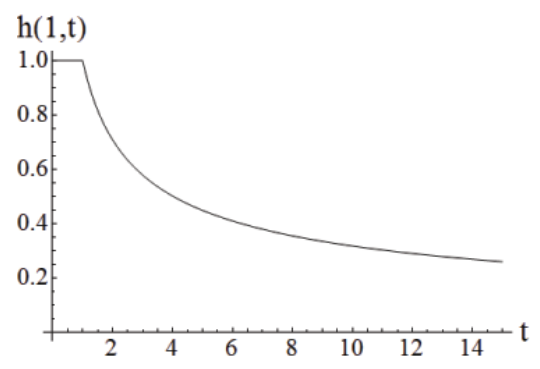

Figure 7. Graph of $h(1, t)$ plotted against drainage time $t$ for $0 \leq t \leq 15$.

We see that a channel is formed in the molasses at time $t=1$. The rate of increase of the width of the channel decreases as $t$ increases. Channelling of the wash water is sometimes observed where water flows through part of the molasses layer and does not wash the remainder. This type of instability is the main consideration motivating the work done in $[5,7,10]$. We also see from Figure 6 that after time $t=15$, about $25 \%$ of the molasses layer remains at the wall of the centrifuge.

Because of the slow rate of removal of the molasses from the crystal for $t>1$ methods of enhanced molasses removal were analysed. 


\section{Enhanced Molasses Removal}

The methods used to enhance the removal of the molasses all reduce the viscosity, $\mu$, of the molasses. The characteristic drainage time, $T$, defined by (2.9) is proportional to $\mu$ and will be reduced when $\mu$ is reduced.

A method which is used to enhance molasses removal is water spraying. The water diffuses into the molasses which is diluted and its viscosity is reduced. Since the diffusion constant has dimensions of $L^{2} / T$ we choose the characteristic time, $T_{D}$, for water diffusion into the molasses to be $T_{D}=h_{0}^{2} / D_{W}$, where $h_{0}$ is the initial thickness of the molasses layer on the crystals and $D_{W}$ is the diffusion constant for diffusion of water into the molasses. Now

$$
h_{0}=2 \times 10^{-4} \mathrm{~m}, \quad D_{W}=5 \times 10^{-10} \mathrm{~m}^{2} \mathrm{~s}^{-1},
$$

and therefore, $T_{D}=80 \mathrm{sec}$. Since the characteristic time for the molasses to drain is $T=25 \mathrm{sec}$, water takes longer to diffuse into the molasses than the time taken for the molasses to drain.

Hydrogen peroxide could also be sprayed on the molasses to bleach the molasses to a lighter colour. The hydrogen peroxide will also remove the molasses in the same way as water. The characteristic diffusion time for hydrogen peroxide to diffuse into the molasses is assumed to be the same as for water.

A third way to enhance molasses removal is by spraying with steam. The molasses is heated by the steam which reduces the viscosity. The characteristic time for heat to diffuse into the molasses is $T_{H}=h_{0}^{2} / D_{H}$, where $D_{H}$ is the diffusion constant for the diffusion of heat into the molasses. Now, $D_{H}=k / \rho c_{p}$, where $k$ is the coefficient of thermal conductivity, $\rho$ is the density of the molasses and $c_{p}$ is the specific heat at constant pressure of the molasses. Now,

$$
k=0.4 \mathrm{~J} \mathrm{~m}^{-1} \mathrm{~s}^{-1} \mathrm{~K}^{-1}, \quad \rho=2 \times 10^{3} \mathrm{~kg} \mathrm{~m}^{-3}, \quad c_{p}=3 \times 10^{3} \mathrm{~J} \mathrm{~kg}^{-1} \mathrm{~K}^{-1}
$$

and therefore $T_{H}=0.6$ sec. Since $T_{H} \ll T$, spraying with steam is a practical way to enhance molasses drainage and is a much better way than diluting with water or hydrogen peroxide. The temperature dependence of the viscosity of molasses is given by [2]

$$
\mu=\mu_{0} /\left[\frac{K-273.15}{K^{2}}\right]^{4.45},
$$

where $\mu_{0}$ is a constant and $K$ is the absolute temperature. The ratio of the characteristic time for the drainage at $100^{\circ} \mathrm{C}$ to the characteristic time at $60^{\circ} \mathrm{C}$ is

$$
\frac{T\left(100^{\circ} \mathrm{C}\right)}{T\left(60^{\circ} \mathrm{C}\right)}=\frac{\mu\left(100^{\circ} \mathrm{C}\right)}{\mu\left(60^{\circ} \mathrm{C}\right)}=0.283 .
$$

But for $t>1$, the volume $V$ of molasses remaining is, from (5.1),

$$
V=\frac{2}{3}\left[\frac{T}{t}\right]^{\frac{1}{2}},
$$

where $t$ is the unscaled time and therefore

$$
\frac{V\left(100^{\circ} \mathrm{C}\right)}{V\left(60^{\circ} \mathrm{C}\right)}=\left[\frac{T\left(100^{\circ} \mathrm{C}\right)}{T\left(60^{\circ} \mathrm{C}\right)}\right]^{\frac{1}{2}}=0.53 .
$$




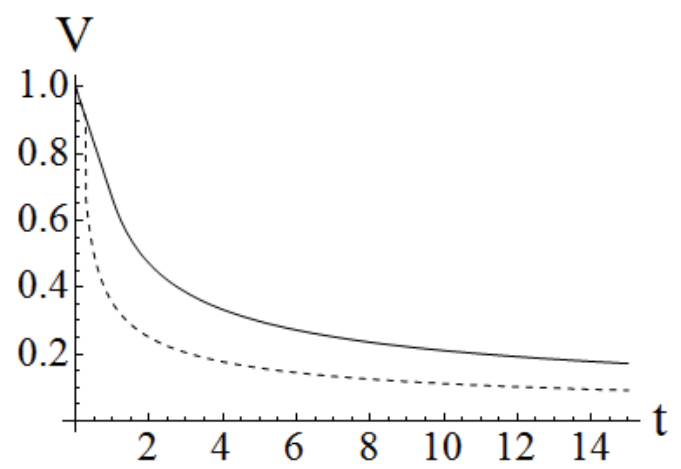

Figure 8. Graph of the volume, $V$, of molasses remaining plotted against time $t$ at $T=60^{\circ} \mathrm{C}$ and $T=100^{\circ} \mathrm{C}$.

In Figure 8 the volume of the molasses remaining at $60^{\circ} \mathrm{C}$ and $100^{\circ} \mathrm{C}$ are plotted against the scaled time $t$ for $60^{\circ} \mathrm{C}$ for $t>1$. We see that spraying by steam reduces by about half the volume of the molasses remaining.

We conclude that heating the molasses by spraying with steam is a better way to enhance the molasses removal than by diluting the molasses by spraying it with water or hydrogen peroxide. Spraying with hydrogen peroxide of course has the other use in the manufacturing process of bleaching the molasses to a lighter colour.

\section{Conclusions}

The characteristic time, $T$, for the drainage of molasses, defined by (2.9), was calculated to be $25 \mathrm{sec}$ and is within the correct order of magnitude. Equation (2.9) is useful because it shows how the parameters $\mu, \Omega$ and $R$ can be varied to control the removal of the molasses from the crystals. The characteristic time does not depend on the permeability of the massecuite, the value of which would be difficult to estimate accurately. The permeability would occur in drainage models formulated in terms of Darcy's law for flow through a porous medium.

The spraying of hydrogen peroxide is used to bleach the molasses. The diffusion time for hydrogen peroxide into the molasses is about $80 \mathrm{sec}$. The molasses layer therefore needs to be kept for several minutes for the hydrogen peroxide to diffuse into the molasses. The hydrogen peroxide will also remove the molasses from the crystals in the same way as water.

We found that channels can occur in the bed of crystals at the outer wall of the centrifuge. This is due to the draining of the molasses from the crystals. Channelling is sometimes observed when wash water flows through part of the bed and does not wash the remaining part.

Washing by water was found not to be a good way to enhance the removal of molasses because the characteristic time for diffusion of water into the molasses is about $80 \mathrm{sec}$. Also the spraying with water dissolves some of 
the sugar crystals leading to losses in production. By far a better way to remove the remaining molasses is to heat the molasses by spraying with steam. The characteristic time for the diffusion of heat into the molasses is about 0.6 sec. Spraying with steam can reduce by about half the volume of the molasses remaining on the crystals.

\section{Acknowledgements}

This problem was submitted by the Sugar Milling Research Institute, Durban, to the Ninth Mathematics in Industry Study Group in South Africa which was held at the African Institute for Mathematical Sciences, Muizenburg, Cape Town, from 9 to 13 January 2012. We thank the African Institute for Mathematical Sciences, the Hermann Ohlthaver Trust and the Science Faculty of the University of the Witwatersrand, Johannesburg, for financial support. We also owe our thanks to Matthew John Woolway, University of the Witwatersrand, Johannesburg, for his assistance in compiling this paper. We thank the referees for their valuable comments and suggestions.

\section{References}

[1] D.J. Acheson. Elementary Fluid Dynamics. Clarendon Press, Oxford, 1990.

[2] B. Barker. Massucuite consistency - A brief review. Technical report, Sugar Milling Research Institute, 2004.

[3] J. Bullen and J.M. de Bruijn. Impact of the centrifugal speed of rotation on the quality of white sugar crystals. Zuckerindustrie, 129:738-741, 2004.

[4] A.K. Davidenko, V.I. Grechanichenko and A.I. Tkachenko. Development of centrifuge construction for the sugar industry. Chemical and Petroleum Engineering, 40:450-454, 2004. http://dx.doi.org/10.1023/B:CAPE.0000047665.33530.59.

[5] N. Fraysse and G.M. Homsy. An experimental study of rivulet instabilities in centrifugal spin coating of viscous Newtonian and non-Newtonian fluids. Phys. Fluids, 6:1491-1504, 1994. http://dx.doi.org/10.1063/1.868263.

[6] H.P. Greenspan. The Theory of Rotating Fluids. Cambridge University Press, Cambridge, 1968.

[7] G.M. Homsy. Viscous fingering in porous media. Ann. Rev. Fluid Mech., 19:271311, 1987. http://dx.doi.org/10.1146/annurev.fl.19.010187.001415.

[8] D.D. Joseph, J. Wang, R. Bai, B.H. Yang and H.H. Hu. Particle motion in a liquid film rimming the inside of a partially filled rotating cylinder. J. Fluid Mech., 496:139-163, 2003. http://dx.doi.org/10.1017/S0022112003006451.

[9] L.M. Jullienne. Washing sugar in batch A-centrifugals. Proc. SASTA, pp. 42-43, 1983.

[10] A. Lindner, D. Bonn, U.C. Poiré, M.B. Amar and J. Meunier. Viscous fingering in non-Newtonian fluids. J. Fluid Mech., 469:237-256, 2002. http://dx.doi.org/10.1017/S0022112002001714.

[11] M. Saska. Reducing sugar colour by washing and bleaching with dilute hydrogen peroxide. Int. Sugar J., 109:616-622, 2007. 